\title{
Oberflächenprozesse in der Geomorphologie - Ansätze und methodische Probleme
}

\author{
Hartmut Leser, Basel
}

Die Landformen haben den Menschen schon immer interessiert, weil sie - z.B. bei der Nutzung des Lebensraumes - «überwunden» werden mussten. Auch geomorphologische Prozesse waren dem siedelnden und wirtschaftenden Menschen frühzeitig bekannt, vor allem dann, wenn man sie als «Naturgefahr» wahrnahm wie Bergstürze, Küstenabbrüche, Flussufererosion oder Dünenwandern. Die Auseinandersetzung geschah direkt, wissenschaftlich unreflektiert und nur auf die Problemlösung bedacht.

Trotzdem ist die Geomorphologie eine eher junge Wissenschaft - sie erwuchs im 19. Jahrhundert aus der Geologie, die einen Bereich «Dynamische Geologie» kannte, was damals aktuelle Prozesse bedeutete. «Dynamische Geologie» im weiteren Sinne würde natürlich auch Erdkrustenbewegungen miteinschliessen, also Tektonik. Im Zeitalter der Interdisziplinarität und Transdisziplinarität haben sich jedoch die Koordinaten verschoben: die strenge Abschottung von Geologie und Geomorphologie hebt sich allmählich auf - das Sachproblem steht im Mittelpunkt. Es ist oft nur lösbar, wenn noch andere Fachwissenschaften herbeigezogen werden wie z.B. Sedimentologie, Mineralogie, Geochemie.

Innerhalb der Geomorphologie haben sich inzwischen massive methodologische und methodische Entwicklungen vollzogen, die an dieser Stelle noch nicht einmal angedeutet werden können. Geblieben ist zunächst einmal die Landform, also die «Erdoberflächenform», zu der das Verständnis der Prozesse gehört. F. AHNerT (2003: 13) diskutierte die Begriffsproblematik ausführlich und meinte zu Recht, dass dem eindeutigen, auch international verständlicheren Begriff «Landformen» (landforms) der Vorzug zu geben ist gegenüber den «ungefügeren und umständlicheren» Begriffen «Erdoberflächenformen» oder «Reliefformen der Erde» in der deutschen Sprache. Die Prozesse waren lange Zeit Hauptgegenstand der Geomorphologie: Formen entdecken und beschreiben, aber auch die Prozesse - zunächst nur «deutend» (also nicht messend) - darzustellen. Allein die geomorphologische Prozessforschung mutierte inzwischen zu einem ganz weit gefächerten Gebiet, das nicht nur nach der Art der Prozesse fragt, sondern auch nach den Randbedingungen, dem zeitlichen Ablauf, der Intensität sowie nach dem Wirkungsgrad. Durch fächerübergreifende Sichtweisen schliesst die Erforschung der «Randbedingungen» inzwischen (und wieder) erdgeschichtliche (geologisch-tektonische) Dimensionen ebenso ein wie Klima, Vegetation, Bodenentwicklung oder den Wasserhaushalt. Man könnte von einem «geomorphologisch-landschaftsökologischen Ansatz» sprechen. Er ermöglicht eine holistische Form-Prozess-Umwelt-Kennzeichnung. Kurzum: Landformen und formbildende Prozesse werden heute in einem quasi allumfassenden Kontext erfasst, zu dem - je länger um so mehr - auch der Mensch gehört. Die Nutzungsansprüche einer wachsenden Erdbevölkerung können auch am Georelief und an den formbildenden Prozessen nicht mehr vorbeigehen.

Was soll damit gesagt werden? Es geht um Vielfalt der in die geomorphologische Betrachtung einbezogenen Sachverhalte, aber auch um die Vielfalt der Betrachtungs- und damit der Erforschungsperspektiven. Daraus resultierte eine Methodenvielfalt, die kaum noch überschaubar ist.

Ähnlich anderen Fachwissenschaften begab sich auch die Geomorphologie in die Spezialisierung hinein - «Datenjagd» und methodische Spitzfindigkeiten an immer kleineren Gegenständen oder gleich im Labor realisiert liessen Grundsätzliches aus dem Blick geraten: Makroformenerfassungen spielten kaum noch eine Rolle (mit Verlust der traditionellen Verbindung zur Geologie) und die real existierenden Unschärfen der Formgestalten und der chaotisch verlaufenden Prozesse wurden ignoriert.

Eine Art von Selbstbesinnung, nicht zuletzt motiviert durch ein immer üblicheres fächerübergreifendes Zusammenarbeiten, rückt wieder Form und Prozess in allen Betrachtungsdimensionen (von der topischen bis zur geosphärisch-globalen) in den Mittelpunkt des geomorphologischen Interesses. Dabei wurden mit einem Male jene theoretischen und methodischen Defizite deutlich, um deren Beseitigung man sich - inzwischen fächerübergreifend - bemüht. Diese Grundsatzproblematik wird im Einleitungsbeitrag von RiCHARD DiKaU «Oberflächenprozesse - ein altes oder ein neues Thema?» aufgegriffen. Er zeigt zunächst einmal die Vielfalt der geomorphologischen Sichtweisen auf die Erdoberfläche auf und weist - in der Landschaftsökologie selbstverständlich - auf die Multiskaligkeit geomorphologischer Systeme hin. Unter Verweis auf eine weit entwickelte interdisziplinäre geowissenschaftliche Methodik müssen die traditionellen Beziehungen zu Geologie, Mineralogie, Geochemie und Bodenkunde 
wiederbelebt werden. Daraus resultiert eine massive Verbreiterung des Methodenspektrums, das aus einer Disziplin allein heraus nicht mehr anzubieten ist. Das bedeutet aber auch eine allseitige Überwindung fachdisziplinärer Eigenheiten, die sich vor allem in den Erdwissenschaften breit gemacht hat.

Die auf den Artikel Richard DiKau folgenden Beiträge des Heftes sind eine Art «Methodensplitter». Sie demonstrieren an verschiedenen geomorphologischen Sachverhalten, mit welchen Ansätzen und Arbeitsweisen heute an den Landformen und an den formbildenden Prozessen gearbeitet werden kann (Abb. 1). Die Ansätze sind (meist) integrativ und die Methoden vom technischen Fortschritt bei Fernerkundung, Geographischen Informationssystemen (GIS), geophysikalischen Methoden, aber auch bei den klassischen Feldmethoden, bestimmt. Zu Letzteren: Es kam, angesichts der faszinierenden Möglichkeiten der Technik, zu einer Vernachlässigung der Feldmethoden, wobei übersehen wurde, dass Technik sich nur dann sinnvoll einsetzen lässt, wenn Ansatz und Methode mit dem Gelände, also den Landformen und den dort real ablaufenden Prozessen, in einem Kontext stehen. Man übersah auch, von Ausnahmen abgesehen, die methodischen Möglichkeiten der geomorphologischen Kartierung, deren methodologische Bedeutung für die Skalenproblematik allmählich wieder erkannt wird, worauf RiChaRD DiKAU in seinem Beitrag ebenfalls hinweist.

Einige dieser Gedanken werden z.B. in dem Beitrag von Nora Jennifer Schneevoigt \& Lothar Schrott aufgenommen. Zu Recht verweisen sie auf F. AHNERT (1994), der vom geomorphologischen System ausgeht, und auf R. Chorley \& B. Kennedy (1971), die für die Physiogeographie das Kaskadensystem kreierten. Diese beiden fundamental wichtigen Titel blieben lange Zeit wenig beachtet. Aber genau sie bieten heute, für den neuen Stand der technischen Forschungsmöglichkeiten, den grundlegenden theoretischen Hintergrund. Er wird, wie im Beitrag gezeigt, durch eine Kombination von GIS und Fernerkundung nicht nur in Wert gesetzt, sondern er macht den Einsatz dieser Methoden erst plausibel. Andererseits machen diese Methodenkombinationen es erst möglich, schwer oder gar nicht zugängliche Hochgebirgsgebiete geomorphologisch exakt darzustellen und deren Prozesssystem zu definieren. Für einen Abschnitt des Reintals im Wettersteinmassiv der Bayerischen Alpen wird dies überzeugend nachgewiesen.

Stärker prozessbezogen ist der Beitrag von LOTHAR Schrott, Joachim Götz, Martin Geilhausen \& David Morche. Auch hier geht es um das Hochgebirge, jedoch um systemanalytisch definierte Sedimenttransporte (Eintrag, innergebietliche Umlagerung, Trans- fer [«Durchsatz»] und Speicherung) in einem alpinen Einzugsgebiet (wiederum das Reintal). Wie im vorhergehenden Beitrag wird wieder mit einer Methodenkombination (Orthofotoauswertung, Vermessung, GIS) gearbeitet. Die Massenmengen werden Ziel- und Quellgebieten zugewiesen, so dass für die einzelnen geomorphologischen Raumeinheiten Bilanzwerte definiert werden können und mit einem Male deutlich wird, dass die Remobilisierung von Sediment ein bisher unterschätzter Prozess der Fluvial- und Hangsysteme war.

Im Beitrag von Rainer Bell, Jan-Erik Kruse, AleJandro Garcia, Thomas Glade \& Andreas Hördt geht es ebenfalls um Materialtransporte, hier jedoch um die klassischen gravitativen Massenbewegungen. Früher waren sie als «Naturkatastrophe», z.B. als Bergsturz, interessant. Mit dem allgemein zunehmenden Flächenanspruch (speziell durch Siedlungsflächen) gerieten auch sie in das Interessensfeld der Öffentlichkeit. Durch die Weiterentwicklung des geowissenschaftlichen Handwerkszeuges werden sie nun nicht nur durch Kartierung oder Bohrung erkundet, sondern mittels Fernerkundung, GIS und geophysikalischen Methoden oder mit Massenbewegungsmodellen dargestellt. Weil für den Ablauf solcher Prozesse die Kenntnis der Gleitflächenzustände erforderlich ist, muss in den Untergrund «hineingeschaut» werden. Dafür steht inzwischen ein breites Spektrum an geophysikalischen Methoden zur Verfügung, von denen der Beitrag die 2D-Geolektrik an einem Beispiel vom Rand der Schwäbischen Alb vorstellt.

Die Bodenerosion ist ebenfalls ein traditionelles Thema der Angewandten Geomorphologie und Landschaftsökologie. Auch hier haben sich die Methoden gewandelt, wenngleich die klassische Schadenskartierung immer noch eine bedeutsame Rolle spielt. Besonders bei der Bodenerosion und deren Bekämpfung muss eine zielgerichtete, d.h. vor allem über den Massstab definierte Methodik eingesetzt werden. Im Beitrag von Petra Ogermann, Bernd Hebel, Volker Prasuhn \& RaINER WeISSHaIDINGER werden kurz und bündig die Möglichkeiten und Grenzen von Erfassungsmethoden dargestellt. Die traditionellen Berechnungsverfahren zeigen immer noch deutliche Unterschiede zu den konventionellen, aber sehr aufwändigen Schadenskartierungen, die schlagbezogen durchgeführt werden (müssen). Es kommt sehr auf das eingesetzte Modell an, aber auch auf die Erwartungen, die man an die damit ermittelten Ergebnisse stellt. Wenn jedoch mit flächenhaftem und gesetzlich geregeltem Bodenschutz ernst gemacht werden soll, sind Modelle unumgänglich. Für die Schweiz schlagen die Autoren daher ein «indikatorbasiertes Monitoring» vor, hinter dem eine Kombination von gezielter Schadenskartierung und flächendeckender Modellierung steht. 


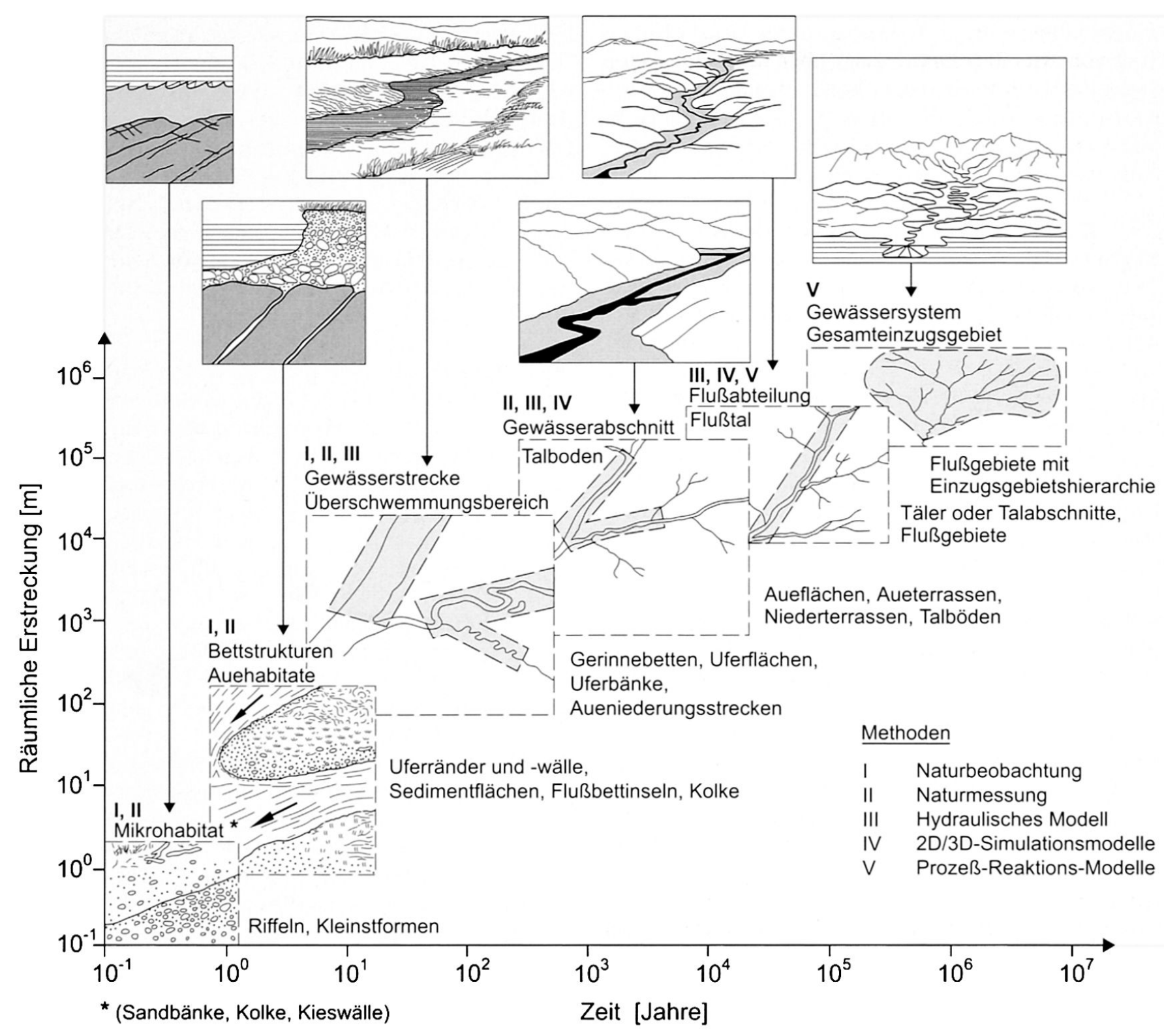

Abb. 1: Raum- und Zeitskalen für die Betrachtung fluvialgeomorphologischer Sachverhalte

Die geomorphologisch interessierenden Gegenstände bei fluvialer Formbildung liegen zwischen dem Mikrohabitat mit Kleinstformen und den Formen eines Großeinzugsgebiets. Die Fluvialformbildung ist also sehr vielgestaltig. Mit ihr sind jeweils spezifische fluvialgeomorphodynamische Prozesse verbunden, die je nach Untersuchungsziel in die Betrachtung Eingang finden. Die methodische Bewältigung (und damit die Datengewinnung an Formen und Prozessen) ordnet sich an zwischen Naturbeobachtungen, Messungen im Feld, hydraulischen Modellversuchen (z.B. «Sandkastenexperimenten» im Labor) und EDV-gestützten Modellsimulationen (nach K. KERN 1994 sowie L.M. HUNZINGER 1998, stark verändert).

Spatial and temporal scales of observation of fluviogeomorphological landform units

Echelles géographiques et temporelles servant à prendre en considération les événements géomorphologiques fluviaux Quelle: LeSER 2003: 241; Entwurf: H. Leser; Graphik: L. BaumanN

Die Fallstudien, die in diesem Heft vorgelegt werden, sollen schlaglichtartig und ausschnitthaft den Stand der geomorphologischen Methodik für die Erkennung der Oberflächenprozesse und ihrer Randbedingungen zeigen. In allen Beiträgen wird deutlich, dass sich nicht nur die Methodik weiterentwickelt hat, sondern dass sehr oft an bewährte traditionelle Verfahren angeknüpft werden muss (z.B. Luftbildauswertung, Kartierung, Beobachtung). Wichtiger erscheint, dass die geomorphologische Theorie z.B. bezüglich Systemansatz, Dimensionsproblem, Formensystematik eigentlich schon vor Jahrzehnten weiter war als der damalige 
Entwicklungsstand der Methodik. Der Übersichtsbeitrag von Richard DikaU zeigt aber auch, dass darin noch ein weiteres, grosses Potenzial steckt, das noch zu nutzen wäre. Dabei wird man um fächerübergreifende Kooperationen nicht herumkommen. Denen hätten sich auch die anderen Mitglieder der «Familie der Geowissenschaften» endlich einmal zu öffnen. Den nach 1980 (und von da an erst allmählich) einsetzenden methodischen Schub für die Geomorphologie konnte es erst geben, als die GIS- und sonstigen EDV-Techniken Allgemeingut wurden. Die Fallbeispiele zeigen, dass diese Möglichkeiten genutzt werden, ohne die Systemtheorie oder ohne die «Theorie der geographischen Dimensionen» zu vernachlässigen. Auch Letztere wurde, und zwar nicht nur in der Geomorphologie, in ihrer Bedeutung erst erkannt, als man durch GIS und Rechenmodelle wieder vermehrt auf die Raumdimensionen, also den Massstab («Skaligkeit»), achten musste.

\section{Literatur}

Ahnert, F. (1994): Equilibrium, scale and inheritance in geomorphology. - In: Geomorphology 11, 2: 125140.

Ahnert, F. (2003): Einführung in die Geomorphologie. - 3., aktualisierte und ergänzte Auflage, Stuttgart: Verlag Eugen Ulmer.

Chorley, R. \& B. Kennedy (1971): Physical geography - A system approach. - London: Prentice Hall International.

HUNZINGER, L.M. (1998): Flussaufweitungen - Morphologie, Geschiebehaushalt und Grundsätze der Bemessung. - = VAW Mitteilungen 159, Zürich.

KERN, K. (1994): Grundlagen naturnaher Gewässergestaltung. Geomorphologische Entwicklung von Fließgewässern. - Berlin, Heidelberg, New York: SpringerVerlag.

LESER, H. (2003): Geomorphologie. - 8., völlig neu bearbeitete Auflage. - Das Geographische Seminar, Braunschweig: Westermann Schulbuchverlag GmbH.

Prof. Dr. Dr. h.c. Hartmut Leser, Geographisches Institut, Abteilung Physiogeographie und Landschaftsökologie, Universität Basel, Klingelbergstrasse $27, \mathrm{CH}-$ 4056 Basel, Schweiz.

e-mail: Hartmut.Leser@unibas.ch 